\title{
Edad de la menarquia y su relación con el nivel socioeconómico e índice de masa corporal
}

\author{
M Isabel Hernández ${ }^{1}$, Nancy U nanue ${ }^{1,2}$, Ximena G aete ${ }^{3}$, \\ Fernando Cassorla ${ }^{1}$, Ethel Codner ${ }^{1}$ \\ Age of menarche and its relationship \\ with body mass index and \\ socioeconomic status
}

\begin{abstract}
Background: A decline in the age of menarche was observed from early 1900s to the 1970s. However, it is not known if a further decline ocurred thereafter. Aim: To evaluate the age of menarche in girls from Santiago, Chile and its relationship with body mass index (BMI) and socioeconomic status. Material and Methods: We studied 1302 healthy girls aged 7 to 19 years. Age of menarche was evaluated through a questionnaire to the patient and her parents. Kaplan-Meier curves were used to determine age of menarche and Cox regression analysis was employed to evaluate the effect of the type of school and BMI on the age of menarche. Results: The mean age at menarche was $12.7 \pm 0.04$ years. Girls from public and private schools had their period at $12.5 \pm 0.1$ and $13.05 \pm 0.05$ years respectively. A negative correlation between $\mathrm{z}$ scores for BMI and age of menarche was observed ( $\mathrm{r}-0.3: \mathrm{p}=0.001)$. Girls whose menarche occurred before 11.5 years had higher $\mathrm{z}$ scores for BMI and a larger proportion were overweight, compared to girls who had menarche later. Cox regression analysis showed that after adjusment for BMI, age of menarche was similar in both types of schools. Conclusions: Age of menarche is ocurring three months earlier in girls from public schools, which is associated with higher z scores for BMI. Type of school, a marker of socio-economic status in Chile, affects timing of menarche due to differences in body mass index (Rev Méd Chile 2007; 135: 1429-36).
\end{abstract}

(Key words: Body mass index; Menarche; Social class)

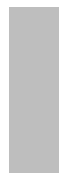
Recibido el 7 de diciembre, 2006. Aceptado el 19 de junio, 2007.
Trabajo financiado parcialmente por el proyecto FONDECYT 1050452.
${ }^{1}$ Instituto de Investigaciones Materno Infantil, Facultad de Medicina, Universidad de Chile. ${ }^{2}$ Servicio de Pediatría, Hospital Clínico San Borja Arriarán. ${ }^{3}$ Servicio de Endocrinología In- fantil, Hospital Roberto del Río. Santiago de Chile.

$\mathrm{D}$ urante el último siglo se ha descrito un adelantamiento progresivo en la edad de la

$\overline{\text { Correspondencia a: Dra. Ethel Codner D. IDIMI, Universidad }}$ de Chile, Casilla 226-3, Santiago, Chile. Tel: 562-4248280. Fax: 562-4247240. E mail: ecodner@med.uchile.cl menarquia. En Estados Unidos de Norteamérica y Europa la edad de la menarquia ha disminuido desde los 14 años, descrito en los años 1900, a los 12,7 años en la década 1970-79, pero no hay un consenso si después de 1970 la edad de la menarquia ha continuado adelantándose o si se 
ha mantenido estable ${ }^{1-4}$. En los países europeos se ha descrito una estabilización en la edad de la primera menstruación, e incluso algunos de ellos describen que ha existido un leve retraso ${ }^{5}$. En la población norteamericana, en cambio, los datos son controvertidos, ya que algunos, como NAHNES III informan un progresivo adelantamiento en la edad de menarquia, mientras que otros no demostrarían variación ${ }^{6,7}$.

En Chile el primer estudio sobre la edad de menarquia fue realizado por la Dra. Eloísa Díaz a fines del siglo 19, que determinó que en las niñas de Santiago la primera menstruación se producía a los 16 años $^{8}$. En 1970 Rona y cols. describieron la menarquia a los 12,6 años en niñas de Santiago? Asimismo, Valenzuela y Avendaño en 1979 evaluaron 2.046 mujeres de estrato bajo y medio-bajo encontrando edad de menarquia promedio de 12,9 años $^{10}$. Otros estudios efectuados en dicho período muestran una edad de menarquia semejante $^{11,12}$ (Tabla 1). Estos datos demuestran que desde fines del siglo 19 hasta la década de 197079 la edad de la menarquia en Chile presentó la misma tendencia secular que en los países del hemisferio norte ${ }^{13}$. En los últimos 20 años existe poca información en Chile sobre la tendencia secular de la menarquia. Recientemente, nosotros evaluamos 758 niñas, pero este estudio tenía un bajo poder para determinar edad de menarquia, ya que sólo 192 niñas de colegios públicos y 8 de colegios privados habían presentado la menarquia ${ }^{14}$.

Uno de los elementos relacionados con el adelanto del inicio de la pubertad ha sido el aumento del peso observado en los niños ${ }^{15}$. En Chile, al igual que en el resto del mundo, también se ha observado un aumento del sobrepeso y obesidad infantil, elementos que podrían estar incidiendo en una edad de menarquia más temprana, y con ello en un inicio de la capacidad reproductiva a menor edad. Por esta razón planteamos la hipótesis que el sobrepeso y el nivel socioeconómico son elementos que influyen en la edad de la menarquia y decidimos evaluar un grupo de niñas provenientes de colegios públicos y privados de Santiago con el objetivo de determinar edad de menarquia y su relación con el tipo de colegio, un buen indicador del nivel socioeconómico, y con el peso corporal.

SuJETOS Y MÉTODO

Se evaluaron un total de 1.302 niñas y adolescentes entre 7 y 19 años. Un grupo de ellas fue reclutado en cinco colegios privados del área oriente de Santiago que corresponden a nivel socioeconómico alto según el Ministerio de Educación (MINEDUC). El segundo grupo provenía de colegios públicos y subvencionados del sector centro y sur de Santiago $(n=11)$ clasificados como de nivel medio-bajo. Al primer grupo lo denominamos Privado y al segundo, Público.

Se realizó encuesta escrita preguntando edad de menarquia (edad, fecha y curso en el que se presentó). Todas las niñas fueron interrogadas por la presencia de alguna patología crónica y se excluyeron aquellas que informaron enfermedades renales, metabólicas, gastrointestinales o cardiopulmonares. Estos datos fueron corroborados por la madre, mediante encuesta enviada al hogar. Se consignó la edad actual (años y meses), y se obtuvo el peso,

Tabla 1. Edad de la menarquia descrita en Santiago en publicaciones de los últimos 40 años

\begin{tabular}{|lcccc|}
\hline Autor & $\begin{array}{c}\text { Año } \\
\text { publicado }\end{array}$ & $\begin{array}{c}\text { Número de niñas } \\
\text { evaluadas }\end{array}$ & $\begin{array}{c}\text { Nivel socio- } \\
\text { económico }\end{array}$ & $\begin{array}{c}\text { Edad menarquia } \\
\text { (años) }\end{array}$ \\
\hline Rona y Pereira9 & 1974 & 354 & Grupo total & 12,6 \\
& & 140 & Alto & 12,96 \\
& 1979 & 214 & Medio-bajo & 12,3 \\
Valenzuela y Avendaño $^{10}$ & 1980 & 6337 & Medio-bajo & 12,75 \\
Patri et al11 $^{11}$ & 1988 & 225 & Bajo & 12,9 \\
Avendaño y Valenzuela $^{12}$ & & Bajo & 12,75 \\
\hline
\end{tabular}


determinado mediante balanza convencional con precisión de $100 \mathrm{~g}$, y la talla, obtenida en un estadiómetro de pared convencional. La antropometría se analizó según la curva NCHS y los resultados se presentan como desviación estándar (DE) para la edad (puntaje Z). La presencia de obesidad se definió como un IMC mayor o igual al percentil 95 de la curva de NCHS 2000 y sobrepeso con un IMC mayor o igual al percentil $85^{16}$. El estudio fue aprobado por el comité de ética del Hospital San Borja Arriarán. A través del respectivo colegio, se solicitó consentimiento informado a los padres de las niñas y sólo participaron en el estudio aquellas cuyos padres dieron autorización por escrito.

Estadística. Se comparó edad de menarquia entre colegios públicos y privados mediante prueba de sobrevida de Kaplan-Meier y prueba de log-rank y la antropometría mediante prueba de $\mathrm{t}$ de Student. Se evaluó correlación entre el Z-IMC y edad de menarquia con prueba de correlación de Pearson. Se evaluó el efecto relativo del tipo de colegio y el Z-IMC sobre la edad de menarquia con análisis de regresión de $\operatorname{Cox}^{17}$. Se definió como menarquia temprana cuando ocurría a los 11,5 años o menos y se comparó la antropometría y la prevalencia de obesidad y sobrepeso en las niñas con menarquia temprana con aquellas que ocurría a edad normal. Se consideró como significativo $\mathrm{p}<0,05$. Los datos se muestran como promedio \pm error estándar (EE).

\section{RESUlTADOS}

Se evaluaron 1.302 niñas escolares, 668 (51,3\%) de colegios públicos y $634(48,7 \%)$ de colegios privados. Las características clínicas según el tipo de colegio se presentan en la Tabla 2. Las niñas de colegios públicos presentaron menor talla, mayor IMC y puntaje $Z$ de peso, y mayor prevalencia de sobrepeso. La edad promedio del grupo total fue de 12,6 $\pm 0,07$ años.

Edad de menarquia (Tabla 2 y Figura 1). El 46,8\% de las niñas evaluadas ya había presentado la menarquia $(\mathrm{n}=634)$. La edad de menarquia para el grupo total fue de 12,7 $\pm 0,04$ años. Las niñas de colegio privado presentaron la menarquia significativamente más tarde que los colegios públicos $(13,0 \pm 0,05$ vs $12,5 \pm 0,1, p<0,001)$. El análisis de la edad de menarquia de las niñas de colegio público en las décadas 1970-79 y 1980-89 (N $=8.822$, Tabla 1) demuestra que en ellas la primera regla ocurría a una edad de 12,8 años, la que es tres meses más tardía que la actual. En cambio las niñas de colegio privado presentan una edad semejante a lo descrito por Rona et $\mathrm{al}^{9}$.

Efecto del peso sobre la edad de menarquia. Se encontró una correlación negativa entre el z-IMC y la edad de menarquia $(r-0,3 ; p=0,001)$. La prueba de regresión de Cox demostró que el ZIMC es un factor determinante de la edad de

Tabla 2. Características clínicas y edad de la menarquia en el grupo total de niñas estudiadas y según su colegio

\begin{tabular}{|lccc|}
\hline & Grupo completo & Colegios públicos & Colegios privados \\
\hline n (\%) & $1302(100 \%)$ & $668(51,3)$ & $634(48,7)$ \\
Edad (años) & $12,6 \pm 0,07$ & $12,5 \pm 0,1$ & $12,7 \pm 0,1$ \\
Talla (Puntaje Z) & $-0,07 \pm 0,02$ & $-0,2 \pm 0,03^{*}$ & $0,04 \pm 0,03$ \\
Peso (Puntaje Z) & $0,1 \pm 0,0$ & $0,4 \pm 0,04^{*}$ & $-0,1 \pm 0,03$ \\
IMC (Puntaje Z) & $-0,2 \pm 0,02$ & $0,6 \pm 0.03^{*}$ & $-0.1 \pm 0.03$ \\
Sobrepeso (\%) & 20,1 & $33,1^{*}$ & 6,6 \\
Obesidad (\%) & 6,9 & $11,9^{*}$ & 1,3 \\
Edad menarquia (años) & $12,7 \pm 0,04$ & $12,5 \pm 0,07^{* *}$ & $13,0 \pm 0,05$ \\
\hline
\end{tabular}

Datos se muestran en promedio \pm error estándar.

*p <0,05 niñas de colegios públicos vs colegios privados;

**p $<0,001$ colegios privados vs públicos según Kaplan-Meier. 
menarquia (EE 0,05, B: 0,35, Wald 43,4, p <0,01), pero que el efecto del tipo de colegio se pierde al ajustar por Z-IMC (EE: 0,09, ß: 0,07, Wald: 0,73, p $=0,4)$. La edad de menarquia en los colegios públicos y privados, ajustada por el Z-IMC, se describe en la Figura 2.
Al comparar el Z-IMC en el grupo de niñas con edad de menarquia temprana, provenientes del total de colegios, se encontró que éstas presentaban mayores Z-IMC y prevalencia de sobrepeso comparada con las que tenían menarquia después de los 11,5 años (Tabla 3).

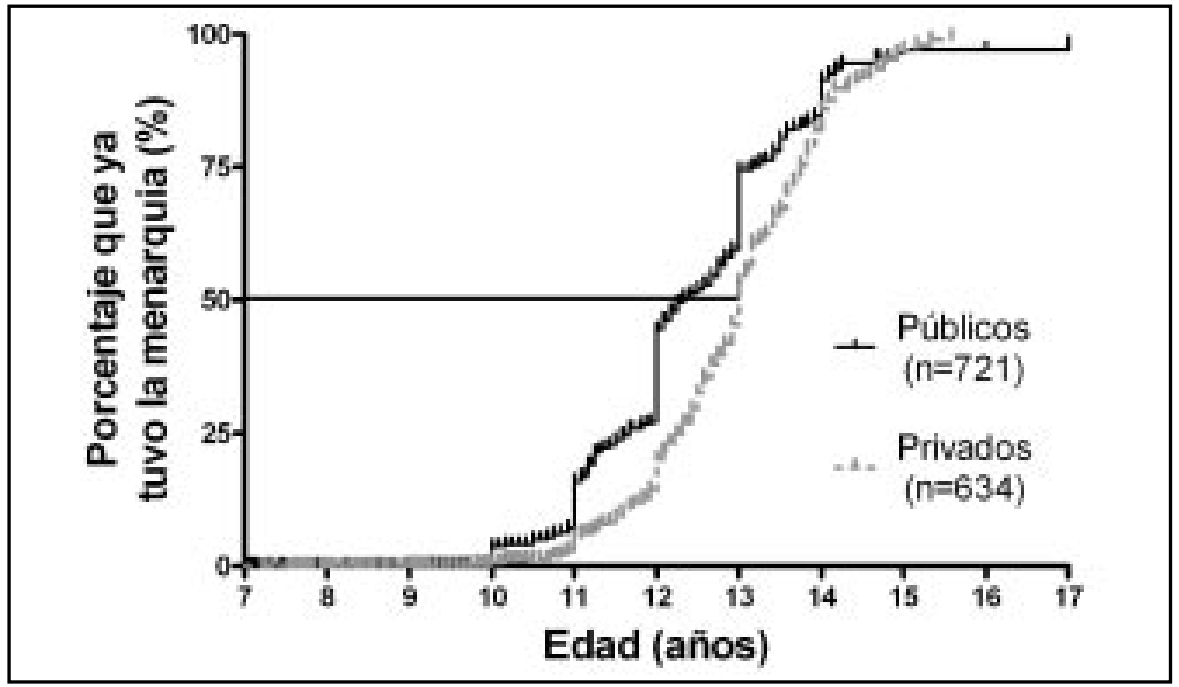

Figura 1. Edad de menarquia según análisis de Kaplan-Meier. La curva muestra el porcentaje de niñas que alcanzaron la menarquia a las diferentes edades. La línea horizontal en el cincuenta por ciento destaca la edad mediana de la menarquia en niñas de colegios públicos y privados ( $\mathrm{p}<0,0001$ prueba de log rank).

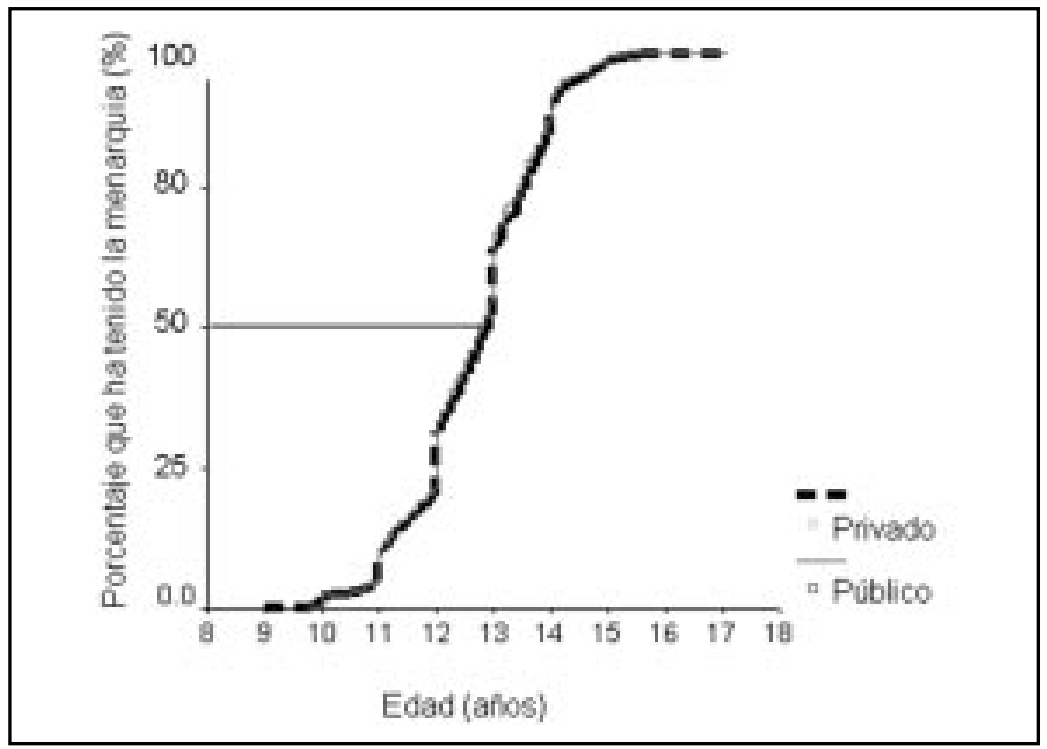

Figura 2. Edad de menarquia según el tipo de colegio corregido por el Z IMC con una prueba de regresión de Cox. La curva muestra el porcentaje de niñas que alcanzaron la menarquia a las diferentes edades. La línea horizontal en el cincuenta por ciento destaca la edad mediana de la menarquia en niñas de colegios públicos y privados. 
Tabla 3. IM C, sobrepeso y obesidad en menarquia a edad temprana o edad normal

\begin{tabular}{|lcc|}
\hline & Menarquia $\leq 11,5$ años & Menarquia $>11,5$ años \\
\hline $\mathrm{n}$ & 150 & 484 \\
IMC (Puntaje Z) & $0,5 \pm 0.01$ & $0,05 \pm 0,03^{*}$ \\
Sobrepeso (\%) & 26,6 & $9,9^{*}$ \\
Obesidad (\%) & 6,0 & $2,5^{\varphi}$ \\
\hline
\end{tabular}

El puntaje Z de IMC (Índice de masa corporal) se muestra como promedio \pm error estándar. *p $<0,001 ;{ }^{\varphi} \mathrm{p}=0,06$.

\section{DisCUSIÓN}

Nuestro estudio efectuado en 1.302 niñas demuestra que presentan la menarquia a los 12,7 años. Esta edad es semejante a lo informado por Rona y cols en el año 1974, que evaluaron 354 niñas del área norte de Santiago con edad entre los 10 y 17 años, y determinaron que ellas presentaban la menarquia a los 12,6 años 9 . Al comparar por nivel socio-económico, las niñas de colegios privados presentaron la menarquia a los 13 años, edad semejante a la publicada por Rona en 1974 (Tabla 1). En el caso de niñas provenientes de colegios públicos observamos que la menarquia se presenta a los 12,5 años, la que es tres meses menor que el promedio de las 8.820 niñas publicadas previamente. También es tres meses más precoz que la publicada por Avendaño y Valenzuela en un estudio longitudinal publicado en $1988^{12}$. Estos datos sugieren que la edad de la menarquia se ha mantenido estable en el nivel socio-económico alto y que ha tenido un adelantamiento de tres meses en los colegios públicos. Esto indica que la edad de la menarquia en Chile se ha comportando en forma semejante a lo observado en diferentes Estados de Estados Unidos de Norteamérica en que se describe un adelantamiento de la menarquia en tres meses en los últimos años ${ }^{6}$.

Recientemente informamos, en un grupo de 754 niñas de Santiago, que la telarquia está ocurriendo a los 8,9 años y que 16\% de las niñas presentan la telarquia antes de los 8 años ${ }^{14}$. El hecho de que la menarquia ocurra 3 años y medio después que la telarquia, sugiere que la pubertad está siendo un proceso lento, de varios años de duración. Además nuestros resultados muestran que el adelantamiento de la pubertad ha afectado fundamentalmente la edad de telarquia y en mucho menor magnitud la edad de primera menstruación.

Los resultados obtenidos en este grupo de niñas demuestran la importancia del peso corporal en la edad de la menarquia. Un tercio de las niñas de colegios públicos presentaron sobrepeso, y $10 \%$ eran obesas, cifra semejante a lo publicado en otros estudios nacionales ${ }^{18}$. Las niñas que presentaron la menarquia antes de los 11,5 años tuvieron mayor IMC, y mayor prevalencia de sobrepeso u obesidad. Además, se observó una correlación negativa entre el z-IMC y la edad de menarquia, y la regresión de Cox confirmó que el IMC es un factor determinante de la edad de la primera menstruación. Por lo tanto, el mayor IMC durante la época infantil se asocia a una menarquia más temprana, llevando a un inicio más temprano de la capacidad reproductiva.

La asociación entre mayor peso corporal y menarquia temprana también conlleva riesgos metabólicos a largo plazo. Estudios en adultos han determinado que a menor edad de la menarquia existe un mayor riesgo de insulino resistencia, dislipidemia, sobrepeso y aumento de la adiposidad abdominal, mayores cifras de presión arterial y mayor frecuencia de intolerancia a la glucosa ${ }^{19-}$ 21. Además diferentes estudios han descrito que la menarquia temprana se asocia a consumo precoz de alcohol y tabaco e inicio temprano de la actividad sexual ${ }^{22-23}$. El peso corporal durante la niñez temprana es un determinante de una pubertad temprana. Estudios recientes han demostrado que niñas con mayor peso a los 5 años ${ }^{24}$, o incluso a $\operatorname{los} 2$ años de $\operatorname{vida}^{25}$ pronostican una 
menarquia o pubertad más temprana. Así mismo, el "Harvard Growth Study", realizó un análisis de los eventos puberales durante los últimos 50 años y demostró una relación entre maduración puberal temprana y relación peso/talla ${ }^{26}$. La razón por la que el mayor peso corporal se asocia con menarquia más temprana se podría explicar porque la adiposidad es un factor importante relacionado con la aceleración de la maduración biológica. Rusell et al demostraron que a mayor adiposidad existe un mayor avance de la edad ósea ${ }^{27}$.

Diversos mecanismos hormonales están involucrados en el avance de la madurez biológica en personas con mayor peso, siendo uno de los más estudiados la secreción de hormonas producidas por el tejido adiposo, o adipocitokinas. La leptina, una hormona que se secreta en forma proporcional a la cantidad de tejido adiposo tiene un rol importante en el inicio puberal ${ }^{28,29}$. Otro mecanismo postulado por el cual un mayor IMC acelera la madurez reproductiva es una disminución de la proteína transportadora de esteroides sexuales (SHBG) secundaria a la insulinorresistencia presente en sujetos con sobrepeso $^{30}$. La disminución de la SHBG produce un aumento de la fracción libre de los esteroides sexuales, lo que puede acelerar la madurez del eje hipotálamo-hipófisis gonadal ${ }^{31}$. Otro mecanismo que puede explicar la aceleración de la pubertad en niños con sobrepeso, es la presencia de mayores niveles de andrógenos suprarrenales tales como dehidroepiandrosterona, y dehidroepiandrosterona sulfato durante los años previos al inicio de la pubertad, los que se metabolizan a testosterona y estrógeno, y por tanto aceleran la maduración ósea $^{32}$. Incluso se ha demostrado que los niños

\section{REFERENCIAS}

1. WYSHAK G, FrISCH RE. Evidence for a secular trend in age of menarche. NEngl J Med 1982; 306: 10335 .

2. Parent as, Teilmann G, Juul A, Skakkebaek Ne, Toppari J, Bourguignon JP. The timing of normal puberty and the age limits of sexual precocity: variations around the world, secular trends, and changes after migration. Endocr Rev 2003; 24: 668-93. obesos al bajar de peso presentan disminución en los niveles de testosterona ${ }^{33}$.

Existen pocos estudios que comparen edad de menarquia según nivel socioeconómico en un mismo país. Sólo dos estudios previos evaluaron este aspecto, uno en población polaca ${ }^{34}$ y otro en un grupo proveniente de Turquía ${ }^{35}$, publicando resultados contradictorios. Nuestro estudio demuestra que las niñas de colegio privado presen$\tan$ su menarquia 6 meses después que las niñas de colegio público, pero que esto se debe a la menor prevalencia de sobrepeso en el primer grupo, sugiriendo que las diferencias de edad de menarquia entre colegios públicos y privados está dada más por el peso de las alumnas que por factores socioeconómicos o étnicos.

En base a nuestros resultados, podemos concluir que se observa un leve adelanto de la edad de la menarquia en las niñas de colegios públicos, pero que éste ha sido de mucho menor magnitud que el adelantamiento de la telarquia. El aumento del peso es un factor determinante en la presencia de menarquia temprana en algunas niñas. Por otra parte el nivel socioeconómico no sería un factor importante por sí mismo, sino que más bien influiría en las diferencias de peso que se observan entre los distintos colegios. Concluimos que la menarquia temprana es otro de los riesgos de la obesidad infantil, y otro motivo para estimular los programas de prevención de sobrepeso.

\section{Agradecimiento}

Agradecemos a los alumnos Claudio Valencia, Jonathan Véliz, Juan Pablo Vildoso por su ayuda con las encuestas.

3. Gluckman PD, Hanson MA. Evolution, development and timing of puberty. Trends Endocrinol Metab 2006; 17: 7-12.

4. De Muinich Keizer SM, Mul D. Trends in pubertal development in Europe. Hum Reprod Update 2001; 7: 287-91.

5. Karlberg J. Secular trends in pubertal development. Horm Res 2002; 57: 19-30.

6. Anderson SE, Must A. Interpreting the continued decline in the average age at menarche: results from two nationally representative surveys of 
U.S. girls studied 10 years apart. J Pediatr 2005; 147: 753-60.

7. Anderson SE, Dallal GE, Must A. Relative weight and race influence average age at menarche: results from two nationally representative surveys of US girls studied 25 years apart. Pediatrics 2003; 111: 844-50.

8. Díaz E. Breves observaciones sobre la aparición de la pubertad en la mujer chilena i las predisposiciones patológicas del sexo. Rev Méd Chile 1888; 16: 289-98, 337-46.

9. Rona R, Pereira G. Factors that influence age of menarche in girls in Santiago, Chile. Hum Biol 1974; 46: 33-42

10. Valenzuela Cy, Avendano A. Antropometría y maduración sexual de estudiantes de una área de Santiago. Bol Oficina SanitPanam 1979; 87: 113-131.

11. Patri AM, Valenzuela CY, Morales CI, Saavedra ZI, Figueroa OL. edad de la menarquia y factores determinantes. Cuad Med Soc 1980; 12-20.

12. Avendaño A, Valenzuela C. Estudio longitudinal de crecimiento y desarrollo: 6 a 20 años de edad. Pediatría (Santiago) 1988; 31: 4-58.

13. Gaete X, Codner E. Adelanto de la pubertad en Chile y el mundo. Rev Chil Pediatr 2006; 77: 456-65.

14. Codner E, Unanue N, Gaete X, Barrera A, MookKanamori D, Bazaes R, et al. Cronología del desarrollo puberal en niñas escolares de Santiago: relación con nivel socio-económico e índice de masa corporal. Rev Méd Chil 2004; 132: 801-8.

15. Kaplowitz PB, Slora EJ, Wasserman RC, Pedlow SE, HERMAN-GidDENS ME. Earlier onset of puberty in girls: relation to increased body mass index and race. Pediatrics 2001; 108: 347-53.

16. Ogden Cl, Kuczmarski RJ, Flegal KM, Mei Z, Guo S, Wei R, Grummer-Strawn LM, Curtin LR, Roche AF, Johnson CL. Centers for Disease Control and Prevention 2000 growth charts for the United States: improvements to the 1977 National Center for Health Statistics version. Pediatrics 2002; 109(1): 45-60.

17. Biro FM, Mcmahon RP, Striegel-Moore R, Crawford pB, Obarzanek E, Morrison Ja, Barton BA et al. Impact of timing of pubertal maturation on growth in black and white female adolescents: The National Heart, Lung, and Blood Institute Growth and Health Study. J Pediatr 2001; 138: 636-43.

18. Albala C, Vio F, Kain J, UauY R. Nutrition transition in Chile: determinants and consequences. Public Health Nutr 2002; 5: 123-8.
19. Remsberg Ke, Demerath EW, Schubert CM, Chumlea WC, Sun SS, Siervogel RM. Early menarche and the development of cardiovascular disease risk factors in adolescent girls: the Fels Longitudinal Study. J Clin Endocrinol Metab 2005; 90: 271824.

20. Must A, Naumova EN, Phillips SM, Blum M, DawsonHughes B, Rand WM. Childhood overweight and maturational timing in the development of adult overweight and fatness: the Newton Girls Study and its follow-up. Pediatrics 2005; 116: 620-7.

21. Kindblom JM, Lorentzon M, Norjavaara E, Lonn L, Brandberg J, Angelhed Je et al. Pubertal timing is an independent predictor of central adiposity in young adult males: the Gothenburg osteoporosis and obesity determinants study. Diabetes 2006; 55: 3047-52.

22. Deardorff J, Gonzales NA, Christopher FS, Roosa MW, Millsap RE. Early puberty and adolescent pregnancy: the influence of alcohol use. Pediatrics 2005; 116: 1451-6.

23. Stice E, Presnell K, Bearman SK. Relation of early menarche to depression, eating disorders, substance abuse, and comorbid psychopathology among adolescent girls.Dev Psychol 2001; 37: 608-19.

24. Davison KK, Markey CN, Birch LL. A longitudinal examination of patterns in girls' weight concerns and body dissatisfaction from ages 5 to 9 years. Int J Eat Disord 2003; 33: 320-32.

25. Dos Santos Silva I, De Stavola Bl, Mann V, Kuh D, HaRdy R ET AL. Prenatal factors, childhood growth trajectories and age at menarche. Int J Epidemiol 2002; 31: 405-12.

26. Cameron N, Demerath EW. Critical periods in human growth and their relationship to diseases of aging. Am J Phys Anthropol 2002; 35: 159-84.

27. Russell DL, Keil MF, Bonat SH, Uwaifo GI, Nicholson JC, McDuffie JR et al. The relation between skeletal maturation and adiposity in African American and Caucasian children. J Pediatr 2001; 139: 844-8.

28. Mantzoros CS, Flier JS, Rogol AD. A longitudinal assessment of hormonal and physical alterations during normal puberty in boys. V. Rising leptin levels may signal the onset of puberty. J Clin Endocrinol Metab 1997; 82: 1066-70.

29. Сhehab FF, Mounzin K, Lu R, Lim ME. Early onset of reproductive function in normal female mice treated with leptin. Science 1997; 275: 88-90. 
30. Holly JM, Smith CP, Dunger DB, Howell RJ, Chard T, Perry LA et al. Relationship between the pubertal fall in sex hormone binding globulin and insulin-like growth factor binding protein-I. A synchronized approach to pubertal development? Clin Endocrinol (Oxf). 1989; 31: 277-84.

31. Nakai Y, Plant TM, Hess DL, Keogh EJ, Knobil E. On the sites of the negative and positive feedback actions of estradiol in the control of gonadotropin secretion in the rhesus monkey. Endocrinology 1978; 102: 1008-14.

32. Ong KK, Potau N, Petry CJ, Jones R, Ness AR, Honour JW ET AL. Avon Longitudinal Study of Parents and Children Study Team. Opposing influences of prenatal and postnatal weight gain on adrenarche in normal boys and girls.J Clin Endocrinol Metab. 2004; 89: 2647-51.

33. Reinehr T, De Sousa G, Roth CL, Andler W. Androgens before and after weight loss in obese children. J Clin Endocrinol Metab 2005; 90(10): 5588-95.

34. Wronka I, Pawlinska-Chmara R. Menarcheal age and socio-economic factors in Poland. Ann Hum Biol 2005; 32: 630-8.

35. Ersoy B, Balkan C, Gunay T, Onag A, Egemen A. Effects of different socioeconomic conditions on menarche in Turkish female students. Early Hum Dev 2004; 76: 115-25. 